\title{
COMMENT
}

\section{Breast feeding and growth trajectories: importance of the time frame of observation}

\author{
Marie Françoise Rolland-Cachera (iD) ${ }^{1}$ \\ Pediatric Research (2020) 87:436-437; https://doi.org/10.1038/s41390-019-0617-z
}

Obesity is an important public health problem and research on the origin of this condition is crucial. The role of early nutrition in predicting later metabolic diseases has generated substantial interest in recent years (the early programming concept or "1000 days," from conception to age 2 years), and numerous studies have confirmed the importance of the early period of life in predicting later health.

Conferring a wide range of favorable effects on health, human milk (HM) is recommended as the optimal food for infants. However, the benefit of breast feeding (BF) for the prevention of overweight and obesity is still questioned. Various reasons can explain discrepancies between studies, particularly the choice of confounding factors ${ }^{1}$ and the length of follow-up.

The longitudinal study by Rodríguez-Cano et al. ${ }^{2}$ describes fat mass (FM) and FM accretion during the first 6 months of life and evaluates FM differences by type of infant feeding. The results show that exclusive/predominant BF promotes higher accretion of FM during the first 6 months of life, as compared to non-exclusive $B F$. These results question whether this pattern is favorable or not.

The different results recorded between short- and long-term follow-up studies can be explained by the frequent changes of growth trajectories observed from early to late childhood. ${ }^{3}$ Typically, body mass index (BMI) increases in the first year of life and then decreases for a number of years before rising again (see Fig. 1). Depending on the child's age at adiposity rebound (AR), which is the age corresponding to the nadir of the BMI curve occurring around the age of 6 years, ${ }^{4}$ the development of body fatness can be very different. Particularly, most infants who are fat by the age of 6-12 months will not stay fat as their BMl will decline until a late AR (case 2). By contrast, lean infants can become overweight after an early AR (case 3 ). These various patterns associated with the age at AR explain the low tracking of BMI during growth.

Whether high or low fatness in early childhood is predictive of later obesity or only transient cannot be concluded from shortterm studies. Early fatness level can be the result of contemporary environmental factors, such as nutrition but does not necessarily predict later health. Various characteristics of HM composition, including type of fatty acids, hormones, vitamins, and oligosaccharide content, are important to determine later health but the macronutrient composition of HM must also be considered. HM is characterized by high fat and low protein contents, which contrast with the well spread belief that high fat intake is associated with obesity and metabolic diseases. The high fat content of $\mathrm{HM}$ is important to cover the high energy needs and rapid neural development during infancy. ${ }^{5}$ It can account for higher fat accretion in early life as observed in the study by Rodríguez-Cano et al. $^{2}$ and may play a beneficial role on future health. In a two decade-long follow-up study examining the association between early nutritional intakes and later development, low fat intake during early life was associated with higher FM and leptin concentration at the age of 20 years. ${ }^{6}$ These results suggest that fat restriction in this critical period of life may activate adaptive mechanisms to facilitate energy storage, thus increasing the susceptibility to later overweight, metabolic diseases, and leptin resistance. Besides, the higher protein consumption in non-breast-fed infants can also promote fatness development as high protein intake in early life is associated with an earlier AR and later overweight. ${ }^{7}$ Consistently, it has been shown that infants who were breast fed for a longer time displayed a later AR and lower fatness at the age of 14 years. $^{8}$ The BMI development pattern, rather than the fatness level in early life, is associated with health risks. The trajectory of body fatness characterized by low level in early life followed by an early AR and later higher fatness development is particularly associated with metabolic diseases, such as diabetes or cardiovascular diseases. ${ }^{3}$

The study by Rodríguez-Cano et al. $^{2}$ shows that exclusive/ predominant $\mathrm{BF}$ promotes higher accretion of $\mathrm{FM}$ during the first 6 months of life but that accretion of FM is higher particularly in the first 3 months. This is consistent with a study conducted in Brazil where infants who were exclusively or predominantly breast fed for the first 4-6 months grew more rapidly than the NCHS reference in weight and length particularly during the first 3 months but appeared to falter thereafter. ${ }^{9}$ By contrast with the hypothesis of an increased fat accretion related to the high fat content of HM, a study that examined breast milk composition and its relationships with growth, reported that higher HM \% fat was associated with lower 3-12-month gains in weight, BMI, and adiposity. ${ }^{10}$ However, this study does not exclude that fatness could have increased during the very first months and faltered thereafter. It is also worth noting that the different results could be related to energy intake, which was not recorded in these studies.

The pattern of higher fat accretion in the first months of life in exclusively BF children as recorded in the study by RodríguezCano et al. could be the first step of a BMI trajectory characterized by early high fatness during the first months of life, followed by longer fatness decrease until the age at AR and lower body fatness in later in life.

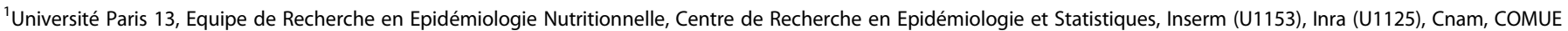
Sorbonne Paris Cité, 93017 Bobigny, France

Correspondence: Marie Françoise Rolland-Cachera (mf.cachera@eren.smbh.univ-paris13.fr)

Received: 5 September 2019 Accepted: 12 September 2019

Published online: 4 November 2019 


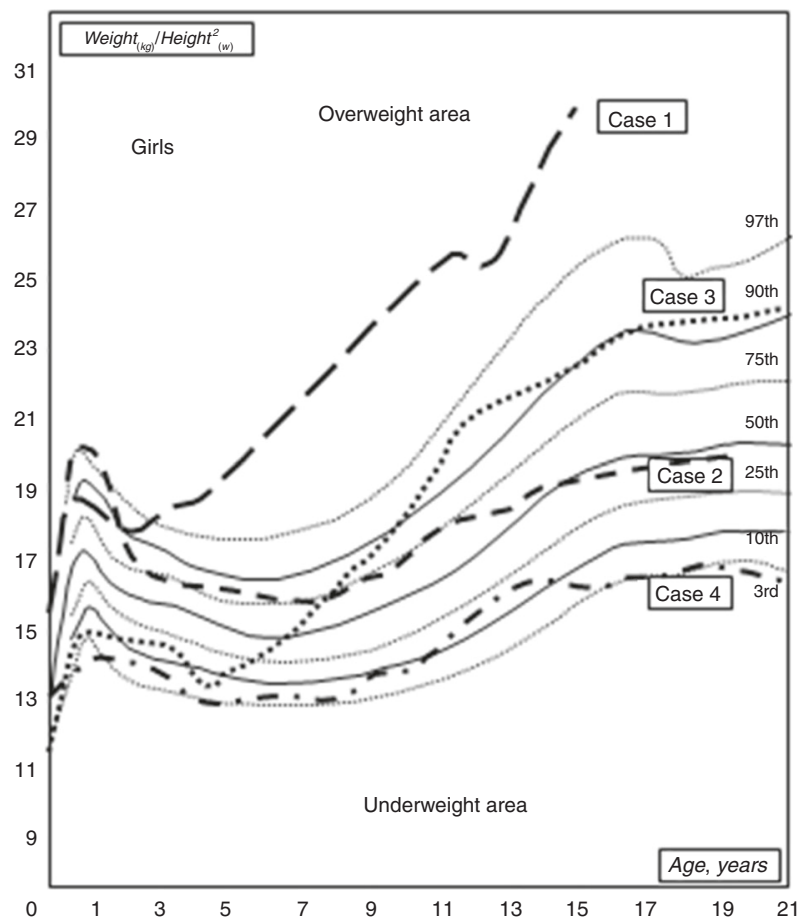

Fig. 1 Four examples of body mass index development plotted against the French reference: Case 1, fat at 1 year, remained fat after an early adiposity rebound ( 2 years); Case 2 , fat at 1 year, did not stay fat after a late rebound ( 8 years); Case 3 , thin at 1 year, became fatter after an early rebound ( 4.5 years); Case 4 , thin at 1 year, remained thin after a late rebound (8 years). From Rolland-Cachera MF, Deheeger M, Guilloud-Bataille M, Avons P, Patois E, Sempé M. Tracking the development of adiposity from one month of age to adulthood. Ann Hum Biol. 1987;14:219-229. Taylor \& Francis Ltd. www.tandfonline.com ${ }^{4}$
Inconsistencies reported in studies examining the association between BF and later fatness emphasize the importance of considering the time frame of observation and the length of follow-up for interpreting the results. Longer longitudinal studies are needed to clarify the effect of HM on growth trajectories and health.

\section{ADDITIONAL INFORMATION}

Competing interests: The author declares no competing interests.

Publisher's note Springer Nature remains neutral with regard to jurisdictional claims in published maps and institutional affiliations.

\section{REFERENCES}

1. Péneau, S., Hercberg, S. \& Rolland-Cachera, M. F. Breastfeeding, early nutrition, and adult body fat. J. Pediatr. 164, 1363-1368 (2014).

2. Rodríguez-Cano, A. M., Mier-Cabrera, J., Allegre-Dávalos, A., Muñoz-Manrique, C. \& Perichart-Perera, O. Higher fat mass and fat mass accretion during the first six months of life in exclusively breastfed infants. Pediatr. Res. https://doi.org/ 10.1038/s41390-019-0542-1 (2019).

3. Rolland-Cachera, M. F. \& Cole, T. J. Does the age at adiposity rebound reflect a critical period? Pediatr. Obes. https://doi.org/10.1111/ijpo.12467 (2019).

4. Rolland-Cachera, M. F. et al. Tracking the development of adiposity from one month of age to adulthood. Ann. Hum. Biol. 14, 219-229 (1987).

5. Uauy, R. \& Dangour, A. D. Fat and fatty acids requirements and recommendations for infants of 0-2 years and children of 12-18 years. Ann. Nutr. Metab. 55, 76-96 (2009).

6. Rolland-Cachera, M. F. et al. Association of nutrition in early life with body fat and serum leptin at adult age. Int. J. Obes. (Lond.) 37, 1116-1122 (2013).

7. Rolland-Cachera, M. F., Akrout, M. \& Péneau, S. Nutrient intakes in early life and risk of obesity. Int. J. Environ. Res. Public Health 13, E564 (2016). pii.

8. Chivers, P. et al. Body mass index, adiposity rebound and early feeding in a longitudinal cohort (Raine Study). Int. J. Obes. (Lond.) 34, 1169-1176 (2010).

9. Victora, C. G., Morris, S. S., Barros, F. C., de Onis, M. \& Yip, R. The NCHS reference and the growth of breast- and bottle-fed infants. J. Nutr. 128, 1134-1138 (1998).

10. Prentice, P. et al. Breast milk nutrient content and infancy growth. Acta Paediatr. 105, 641-647 (2016) 